\title{
Análise microestrutural de argamassa de revestimento com substituição parcial do agregado natural por sedimento do Rio Amazonas
}

Microstructural analysis of coating mortar with partial replacement of natural aggregates by sediment from the Amazon River

\begin{tabular}{|c|c|}
\hline & $\begin{array}{l}\text { Maurílio Gomes-Pimentel (D) } \\
\text { Isaura Nazaré Lobato Paes }\end{array}$ \\
\hline & 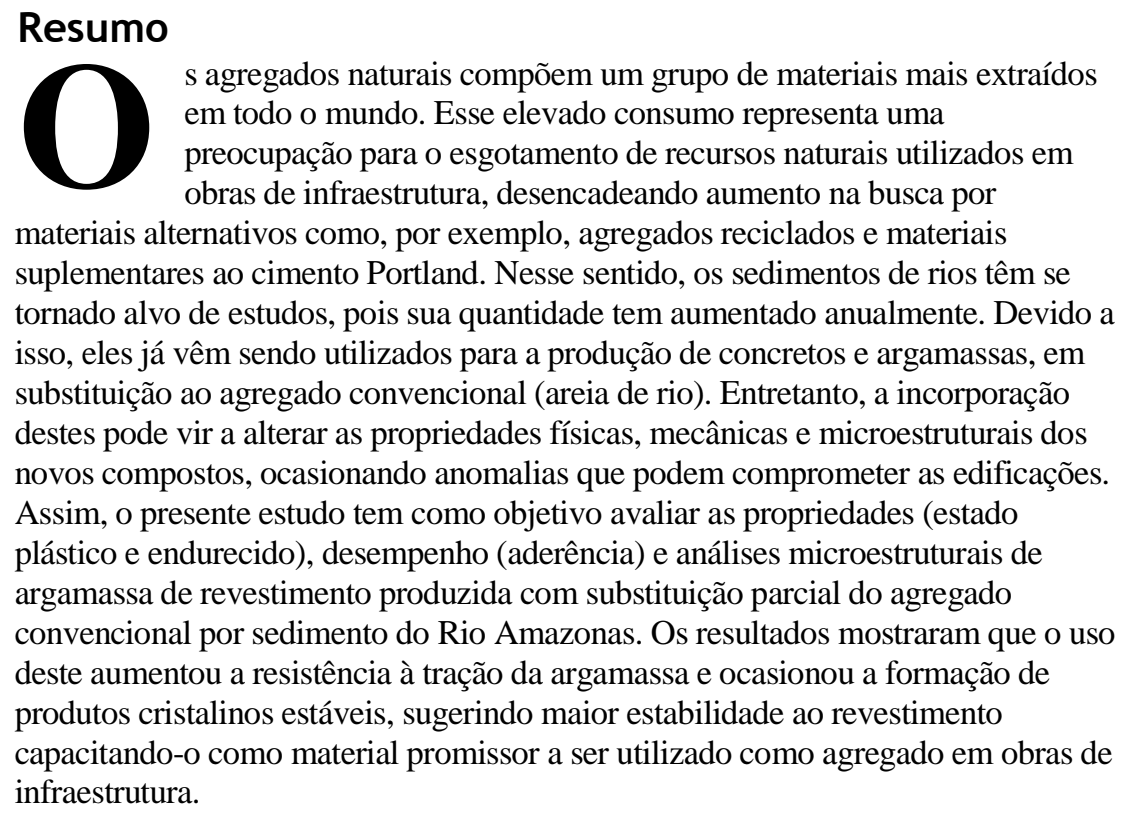 \\
\hline & $\begin{array}{l}\text { Palavras-chave: Areia de sedimento. Argamassa. Desempenho. Microestrutura. } \\
\text { Abstract }\end{array}$ \\
\hline $\begin{array}{r}{ }^{1} \text { Maurílio Gomes-Pimentel } \\
{ }^{1} \text { Universidade Federal do Pará } \\
\text { Belém -PA - Brasil }\end{array}$ & $\begin{array}{l}\text { Natural aggregates are among the of most intensively extracted natural materials } \\
\text { in the world. This high consumption is a great concern in terms of the depletion of } \\
\text { the natural resources used in infrastructure works, which has led to an increase in } \\
\text { the search for alternative materials such as recycled aggregates and } \\
\text { supplementary materials to Portland cement. Hence, the sediments of rivers have } \\
\text { become the target of severalstudies, leadingtheirquality to improveyearly. As a } \\
\text { consequence, river sediments arealready being used in the production of concrete } \\
\text { and mortar, replacing conventional aggregate (river sand). However, their } \\
\text { incorporation can alter the physical, mechanical and microstructural properties of } \\
\text { the new compounds, causing anomalies that can compromise the buildings. Thus, } \\
\text { the goal of this study is to evaluate the properties (plastic and hardened state), } \\
\text { performance (adhesion) and perform a microstructural analysis of coating mortar } \\
\text { produced with partial replacement of conventional aggregate by sediment from the }\end{array}$ \\
\hline $\begin{array}{r}\text { } 2 \text { Isaura Nazaré Lobato Paes } \\
\text { 2Universidade Federal do Pará } \\
\text { Belém -PA - Brasil }\end{array}$ & $\begin{array}{l}\text { Amazon river. The results showed that its use increased the tensile strength of the } \\
\text { mortar and caused the formation of stable crystalline products, suggesting the } \\
\text { coating acquired greater stability, thusmakingriver sediment a promising material } \\
\text { to be used as an aggregate in infrastructure works. }\end{array}$ \\
\hline & Iiment sand. Mortar. Performance. Microestructure. \\
\hline
\end{tabular}




\section{Introdução}

A urbanização e o crescimento populacional global estão alimentando o desenvolvimento das obras de edificações e infraestrutura de transportes, como consequência há aumento significativo na demanda por matéria-prima, como, por exemplo: areia e brita. De fato, a areia e a brita compõem o grupo de materiais mais extraídos no mundo, superando os combustíveis fósseis e a biomassa (UNITED..., 2016). Na maioria das regiões a areia é considerada um bem em comum, ou seja, um recurso aberto a todos, isso faz com que sua extração seja realizada de forma desenfreada, sem considerar as consequências em longo prazo (REGE, 2016). Como resultado, a escassez de areia é uma questão emergente, com grandes implicações sociopolíticas, econômicas e ambientais (SUTHERLAND et al., 2017). Aproximadamente 32 bilhões a 50 bilhões de toneladas de areia são usadas globalmente por ano, majoritariamente para a fabricação de concretos e argamassas (KOEHNKEN, 2018), isso excede o ritmo natural de renovação desses recursos (PEDUZZI, 2014), fazendo com que em meados do século a demanda por areia supere a oferta (SVERDRUP; KOCA; SCHLYTER, 2017).

A escassez de areia já é uma grande realidade em muitas regiões, como na Nigéria, Paquistão e Índia (BENDIXEN et al., 2019), e nessas regiões os altos lucros gerados pelo comércio desse material geralmente levam a conflitos sociais e políticos, incluindo violência, extração ilegal desenfreada e tensões entre as nações. Na índia, por exemplo, a "máfia de areia" é considerada um dos grupos de crime organizado mais poderosos e violentos e centenas de pessoas já foram mortas nas "guerras de areia" (REGE, 2016). Isso tudo tem feito com que a busca por materiais alternativos torne-se uma realidade necessária para suprir a demanda na produção de concretos e argamassas.

Muitos estudos sugerem uma variedade de materiais que podem ser utilizados como elemento suplementar à areia, podendo atender as tendências atuais de desenvolvimento, como a escória de alto forno (ADEGOLOYE et al., 2016), que quando resfriada lentamente fica inviabilizada de ser utilizada como material suplementar ao cimento Portland devido a sua baixa reatividade, mas tem se mostrado promissora para uso como substituto de agregados na construção (GUO et al., 2019). Outros exemplos são o uso de resíduos de rochas trituradas para a produção de minérios metálicos (UNITED..., 2016), rejeitos de mineração de metais (FONTES et al., 2016; YARAGAL; BASAVANA GOWDA; RAJASEKARAN, 2019), resíduos gerados na produção do mármore e granito (KHYALIYA; KABEER; VYAS, 2017), resíduos agroindustriais e concretos reciclados (LEDESMA et al., 2014; ZHAO et al., 2015).

Em virtude do exposto, no que tange à busca de materiais alternativos com aplicações voltadas ao uso como agregados, o presente estudo tem como objetivo avaliar a utilização de um sedimento proveniente do Rio Amazonas, como substituição parcial ao agregado miúdo natural nas propriedades de argamassa de revestimento, no estado plástico e endurecido, e como suas características microestruturais podem vir a influenciar seu desempenho, mais especificamente a resistência de aderência à tração de argamassas destinadas a revestimento externo.

A origem desse material é devida à elevada precipitação e à topografia íngreme da Cadeia Andina (berço do Rio Amazonas), que está sujeita à intensa erosão e carreando enormes quantidades de carga de sedimento para os afluentes do referido rio. Esses sedimentos, quando em suspensão, devido ao transporte fluvial, são parcialmente descarregados no Oceano Atlântico. Porém, cerca de $20 \%$ desse material é depositado na extensão da região que vai da cidade de Óbidos, PA,à cidade de Macapá, AP, formando os chamados "bancos de sedimentos em suspensão". A quantidade dos sedimentos transportados pode chegar a 900.106 t/ano (PARK; LATRUBESSE, 2014; GENSAC et al., 2016).

O uso desse tipo de material, como componentes de argamassas e concretos, vem atraindo pesquisas em nível nacional e internacional. Estudos avaliaram o potencial de substituição (parcial) do agregado natural, e até mesmo do cimento, em tais componentes (ROZIÈREA et al., 2015;LAOUFI; SENHADJIA; BENAZZOUKB, 2016). Os resultados desses estudos revelam que quando o sedimento é tratado termicamente pode ser utilizado como material suplementar ao cimento, por conta da presença de argilominerais hidratados como a caulinita, que geralmente está presente na constituição do material. E quando ele é utilizado como agregado miúdo, melhoram a reologia do concreto. No entanto, apesar de pesquisas comprovarem que esse material (sedimento) pode ser utilizado de forma a obter resultados satisfatórios nas matrizes cimentícias, há casos em que foi reportada a necessidade de se realizar tratamento para eliminação de frações orgânicas e outros poluentes (WANG et al., 2015; BENZERZOUR; AMAR; ABRIAKA, 2017). 
Outros estudos sugerem que haja restrições dos percentuais a serem utilizados, pois materiais contaminantes podem estar presentes nas frações mais finas como chumbo $(\mathrm{Pb})$ (COUVUDAT et al., 2016). Assim, é pertinente a realização de pesquisas que venham a balizar o uso desse sedimento e como ele irá atuar no desempenho e durabilidade dos componentes.

No estado do Amapá (Brasil), por exemplo, há registros da utilização intensa desse material na execução de argamassas de revestimento e assentamento, porém sua aplicação acontece de forma empírica, principalmente no que tange a sua dosagem racional. Nesse sentido, devido à sua granulometria mais fina, em comparação ao agregado natural, a avaliação do desempenho é muito importante, pois o teor de finos (partículas menores que $75 \mu \mathrm{m}$ ) pode influenciar na relação água/cimento e, consequentemente, ocasionar problemas de fissuras por retração plástica, pulverulência, diminuição da estanqueidade e redução da aderência, entre outros. Além disso, a incorporação de materiais não convencionais na produção de concretos e argamassas pode alterar as propriedades físicas, mecânicas e microestruturais dos novos compostos, desencadeando manifestações patológicas que podem vir a comprometer as obras de infraestrutura.

\section{Materiais}

Para a realização desta pesquisa foram empregados os seguintes materiais, a saber: cimento Portland CP IV 32 , com massa específica de $2.720 \mathrm{~kg} / \mathrm{m}^{3}$, massa unitária de $1.190 \mathrm{~kg} / \mathrm{m}^{3}$, área específica de $455,20 \mathrm{~m} / 2 \mathrm{~kg}$ (método Blaine) e resistência à compressão de 19,7 MPa, 24,6 MPa e 36,8 MPa, para as idades de 3, 7 e 14 dias, respectivamente.Um agregado miúdo natural (areia de rio), sedimento proveniente da cidade de Macapá, AP, aditivo plastificante e blocos cerâmicos de vedação. Além desses, utilizou-se água potável com temperatura de $28^{\circ} \mathrm{C}$ e $\mathrm{pH} 6$.

Para a argamassa de revestimento, com traço de referência de 1:5 (cimento: areia, em massa), foi adicionado o aditivo plastificante de fabricação nacional e de composição organo-sintética. Os componentes ativos básicos são resíduos da destilação de resina de pinho e resina de breu, segundo o fabricante. O produto é comercializado na forma líquida, com massa específica igual a $1.000 \mathrm{~kg} / \mathrm{m}^{3}$. O teor de aditivo utilizado na execução das argamassas foi de $4 \times 10^{6} \mathrm{~m}^{3} / \mathrm{kg}$ de cimento. Cabe ressaltar que o proporcionamento de 1:5 foi utilizado por ser, na região metropolitana de Belém, comumente empregado nos revestimentos de fachadas, com variação entre 1:5 a 1:7 (cimento: areia, em massa).

Cabe destacar que o aditivo supracitado só foi adicionado à argamassa de referência (AAN) a fim de ocasionar trabalhabilidade à mistura. $\mathrm{O}$ seu emprego não foi requerido para a argamassa com sedimento (ASD), uma vez que a substituição do agregado natural pelo sedimento (25\%) ocasionou plasticidade à argamassa.

$\mathrm{O}$ agregado natural foi uma areia de rio com massa específica de $2.630 \mathrm{~kg} / \mathrm{m}^{3}$. A areia apresentou como material cristalino unicamente o quartzo, enquanto o sedimento apresentou cristais de caulinita, clinochlore e phlogopite (Figura 1a). Além disso, a granulometria do sedimento foi inferior à da areia (Figura 1b) e sua morfologia é típica de quartzo com superfície porosa em algumas partículas (Figura 1c).

Uma premissa-chave do presente estudo é que o sedimento exibe um elevado teor de $\mathrm{SiO}_{2} \mathrm{em}$ forma de quartzo (Tabela 1, composição determinada por FRX) além de caulinita, sendo o primeiro inerte em matriz cimentícia, e o segundo um argilomineral podendo atuar com os componentes do cimento Portland para formação de novos produtos hidratados ou como carga reforçando a matriz e aumentando a resistência. $\mathrm{O}$ sedimento também apresenta elevado teor de $\mathrm{Al}_{2} \mathrm{O}_{3}$, similar à quantidade encontrada no cimento.

\section{Dosagem das argamassas}

Utilizou-se nesta pesquisa uma argamassa de revestimento de referência (AAN) habitualmente empregada na região metropolitana de Belém, PA, com traço 1:5 (cimento: areia, em massa). A influência da variação dos teores de substituição da areia pelo sedimento (ASD) foi analisada por meio de estudo de dosagem e ensaios de caracterização no estado plástico e ensaios no estado endurecido.

As argamassas foram executadas em uma betoneira de eixo inclinado e a ordem de adição na betoneira foi: agregado, cimento e água, respectivamente, misturando-se completamente em um intervalo de tempo de 5 minutos. Os proporcionamentos (traços) estão representados na Tabela 2. 
Figura 1- Características físicas e morfológica dos agregados - (a) difração de raios X; (b) granulometria dos agregados; (c) microestrutura eletrônica de varredura do sedimento
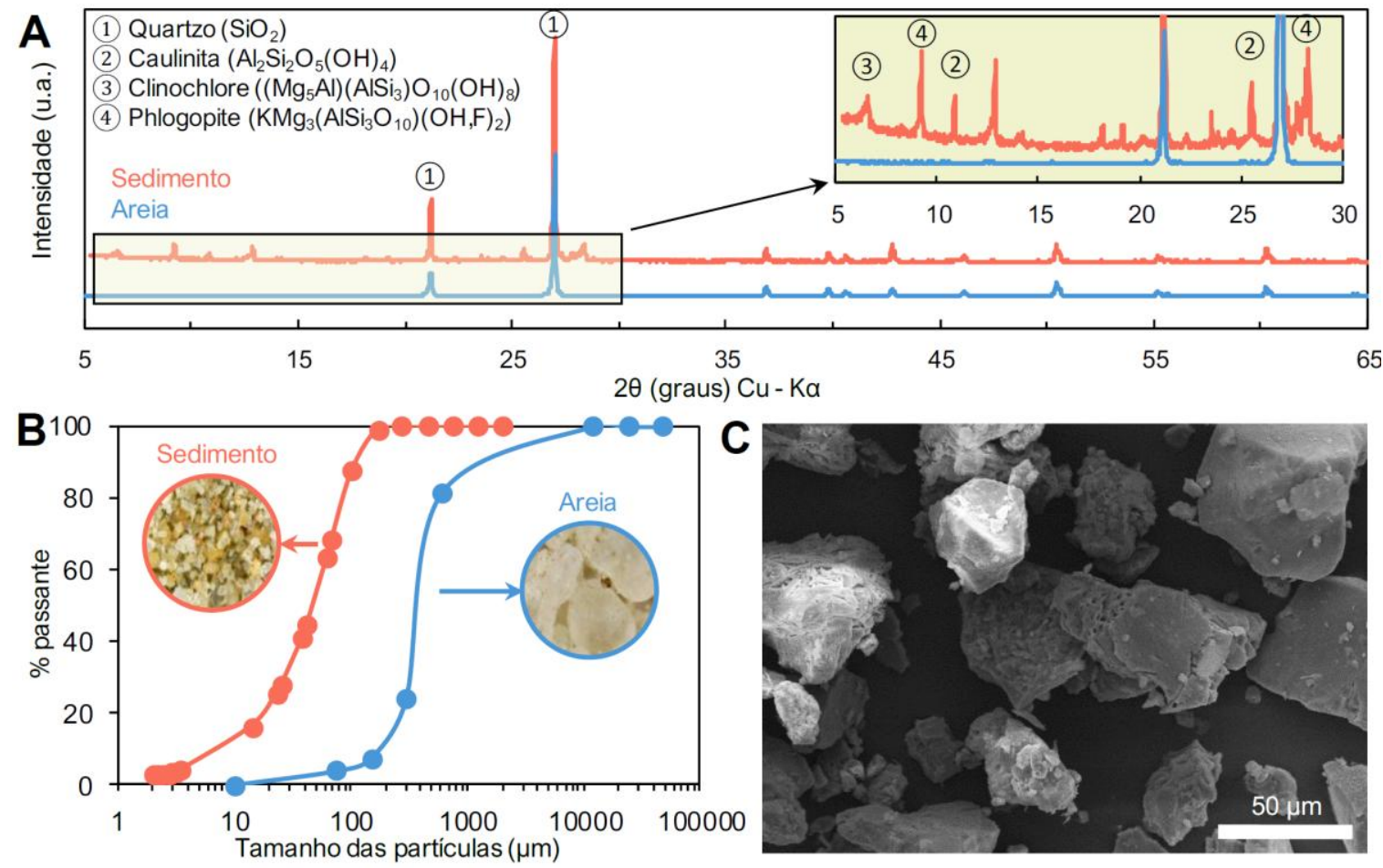

Tabela 1 - Composição química dos materiais - valores em (\%) - PF é perda ao fogo

\begin{tabular}{c|c|c|c|c|c|c|c|c|c|c|c|c}
\hline Material & $\mathbf{S i O}_{\mathbf{2}}$ & $\mathbf{C a O}$ & $\mathbf{M g O}$ & $\mathbf{F e}_{\mathbf{2}} \mathbf{O}_{\mathbf{3}}$ & $\mathbf{A l}_{\mathbf{2}} \mathbf{O}_{\mathbf{3}}$ & $\mathbf{S O}_{\mathbf{3}}$ & $\mathbf{N a}_{\mathbf{2}} \mathbf{O}$ & $\mathbf{K}_{\mathbf{2}} \mathbf{O}$ & $\mathbf{M n O}$ & $\mathbf{P}_{\mathbf{2}} \mathbf{O}_{\mathbf{5}}$ & $\mathbf{T i O}_{\mathbf{2}}$ & $\mathbf{P F}$ \\
\hline Areia & 94,34 & - & - & 3,27 & - & - & - & - & - & - & - & 2,39 \\
Sedimento & 78,87 & 0,89 & 0,67 & 3,14 & 9,48 & - & 1,44 & 1,57 & 0,01 & 0,12 & 0,71 & 3,04 \\
Cimento & 31,00 & 44,83 & 4,02 & 3,51 & 9,70 & 1,21 & 0,18 & 0,90 & - & - & - & 4,65 \\
\hline
\end{tabular}

Tabela 2 - Traços utilizados para confecção das argamassas de revestimento (referência) e com substituição da areia natural por sedimento

\begin{tabular}{c|l|c}
\hline Argamassas & \multicolumn{1}{|c|}{ Traços (em massa) } & Relação água/cimento \\
\hline AAN & $1: 5,0$ (cimento: areia) + adit. plastificante & 0,94 \\
ASD & $1: 1,25: 3,75$ (cimento: sedimento: areia) & 1,02 \\
\hline
\end{tabular}

Antes da moldagem das argamassas para avaliação das propriedades no estado endurecido, foram avaliadas as propriedades no estado fresco, a saber: consistência de acordo com a NBR 13276 (ABNT, 2016), densidade de massa e teor de ar incorporado de acordo com a NBR 13278 (ABNT, 2005a). Já no estado endurecido foram avaliadas propriedades como resistência à tração na flexão e à compressão de acordo com a NBR 13279 (ABNT, 2005b) e absorção de água por capilaridade de acordo com a NBR 9779 (ABNT, 2005c).

\section{Execução de painéis para avaliação de desempenho e características microestruturais das argamassas}

Para a etapa de avaliação de desempenho foram confeccionados seis painéis em alvenaria de bloco cerâmico de vedação (Figura 2a) com dimensões de $(1,20 \times 1,50) \mathrm{m}^{2}$. Posteriormente foi realizada a aplicação da argamassa de chapisco com traço de 1:3 (cimento: areia, em massa) o qual é comumente sugerido em normas de execução de revestimento e amplamente utilizado in situ. Após 14 dias se realizou o lançamento das argamassas de revestimento (AAN e ASD). 
Cabe destacar que os painéis foram confeccionados em área externa do Laboratório de Engenharia Civil da UFPA com objetivo de produzir condições agressivas de intemperismo (altas temperaturas, elevada umidade relativa e chuvas constantes) similares às do clima da região em que foi realizada a pesquisa, conforme mostrado na Figura 2. As condições de umidade e temperatura variaram ao longo do tempo (Figura 2d) até a idade de ensaio (63 dias).

A determinação da resistência de aderência foi realizada nos revestimentos na idade de 63 dias, seguindo os procedimentos descritos na NBR 13528 (ABNT, 2010). Para a realização desta foi empregado um dinamômetro de impacto e, em cada painel, foram realizados 12 arrancamentos perfazendo um total de 72 mensurações. Registrou-se as cargas de ruptura, o diâmetro efetivo do corpo de prova, a espessura do revestimento e os percentuais dos tipos de ruptura.

Para as análises microestruturais, as amostras foram removidas da superfície do painel por meio do uso de uma serra copo diamantada, onde se buscou evitar contaminações. Estas passaram por processo de pulverização para análise de difração de raios X (DRX), pelo método do pó. A análise de DRX foi realizada em um difratômetro de feixe divergente equipado com um goniômetro $\theta-\theta$, um tubo de raios $\mathrm{X}$ de $\mathrm{Cu}$ com radiação de $\mathrm{Cu}(\mathrm{K} \alpha 1=1,540598 \AA)$, um filtro de $\mathrm{Ni} \mathrm{K} \beta$. As condições do instrumento foram as seguintes: 40 $\mathrm{kV}$ e $40 \mathrm{~mA}$; fenda Soller de $2,5^{\circ}$ (feixe incidente e refratado); faixa angular (20) de $5^{\circ}$ a $65^{\circ}$. A coleta dos difratogramas consistiu em obter umpasso angular de $0,02^{\circ}$ e tempo por passo de $0,5 \mathrm{~s}^{\circ}$, fenda divergente de $0,6 \mathrm{~mm}$.

A microscopia eletrônica de varredura das argamassas (MEV) foi obtida por meio de um microscópio Zeiss modelo Sigma-VP, as amostras foram preparadas com extração dos fragmentos e fixação em um suporte de fita de carbono $(\mathrm{C})$ e metalizadas com ouro $(\mathrm{Au})$. Efetivaram-se as análises com utilização deelétrons secundários para obtenção das imagens a $20 \mathrm{kV}$, com uma distância de trabalho de $15 \mathrm{~mm}$. Além disso, foi realizado o mapeamento dos elementos por energia dispersiva (EDS) e calculadas as proporções atômicas $(\mathrm{Ca} /(\mathrm{Si}+\mathrm{Al}))$ e $(\mathrm{Al} /(\mathrm{Si}+\mathrm{Al}))$.

\section{Análise dos dados}

Analisou-se os dados quanto a sua normalidade e sua homocedasticidade. O teste de Shapiro-Wilk mostrou que as amostras apresentam distribuição normal por ensaio $(\mathrm{P}>0,05)$, e o teste de Levene afirma que as variâncias são homogêneas $(\mathrm{P}>0,05)$. A partir dessas apreciações, a análise estatística foi efetivada utilizando-se o teste $\mathrm{T}$ de Student, por haver apenas duas condições experimentais. Assim, os dados foram representados como média aritmética \pm desvio padrão, e se considerou diferença significativa quando $\mathrm{P}<0,05$.

Figura 2 - (a) Bloco cerâmico de vedação; (b) painel com argamassa aplicada; (c) consistência e (d) condições de temperatura e umidade ao longo do tempo do painel até a idade de ensaio

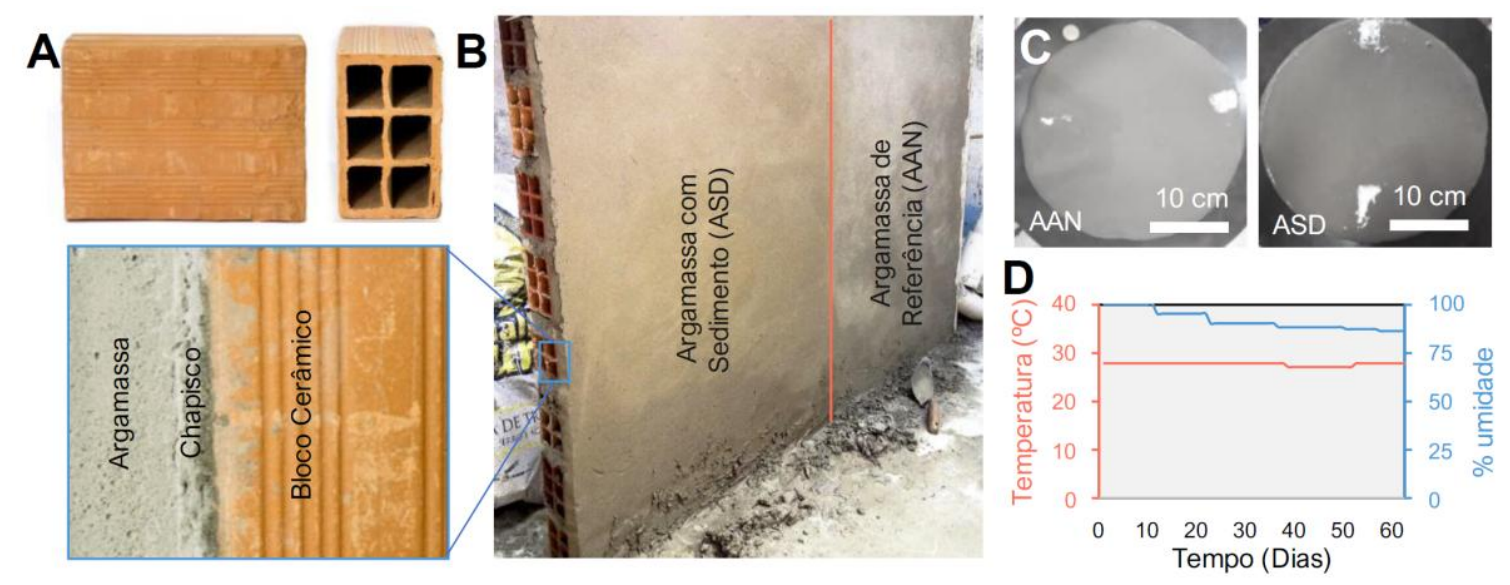




\section{Resultados e discussão}

Como já destacado anteriormente, para a obtenção da plasticidade da argamassa com sedimento não houve necessidade de ser adicionado ao traço qualquer outro material (cal ou aditivo) para a obtenção desta. O sedimento, por si só, já forneceu trabalhabilidade à mistura, possivelmente devido a suas partículas finas propiciarem o chamado efeito "liga" muito conhecido, por exemplo, quando da utilização de materiais saibrosos as argamassas. Esse fato é interessante de ser observado, uma vez que em uma análise de custo (que não foi objeto desta pesquisa) esse parâmetro pode vir a ser relevante (Figura 3a, $\mathrm{T}_{(9)}=1,942 ; \mathrm{P}=0,08$ ).

A análise da consistência mostra que, com a adição de $25 \%$ do sedimento, houve um incremento desta (espalhamento da argamassa), uma vez que houve necessidade de se adicionar maior quantidade de água para obtenção da trabalhabilidade. Apesar desse fato, a argamassa com sedimento obteve maior densidade de massa, em comparação à argamassa de referência (Figura 3b, $\mathrm{T}_{(4)}=14,779 ; \mathrm{P}<0,001$ ). Possivelmente isso ocorre devido à maior quantidade de partículas finas inerentes à granulometria do sedimento e que acabam por preencher internamente os espaços vazios (interstícios) da argamassa aumentando tal propriedade (PIMENTEL; PAES, 2019).

Já com relação ao teor de ar incorporado, este foi maior para a argamassa sem o sedimento (Figura 3c, $\left.\mathrm{T}_{(4)}=19,595 ; \mathrm{P}<0,001\right)$. Isso pode ter acontecido em virtude do uso de aditivo na argamassa com $100 \%$ de agregado natural. Na prática, esse fato é bastante favorável, uma vez que as argamassas aditivadas necessitam além do controle do teor do aditivo e do tempo de mistura, de forma que o ar incorporado à argamassa não venha a influenciar negativamente nas propriedades mecânicas da argamassa, bem como no seu desempenho.

Os resultados das propriedades avaliadas mostram a relação direta entre a interação do agregado natural e o sedimento. A mescla entre estes possivelmente gerou uma microestrutura mais densa com diminuição do volume de vazios e, consequentemente, com aumento das propriedades mecânicas. A resistência à compressão axial (Figura 4a) e a resistência à tração na flexão (Figura 4b) foi maior para a argamassa com sedimento.

Já com relação às características de absorção e velocidade de penetração de água, sabe-se que estas também são alteradas, entre outros, pela composição e proporcionamento entre os materiais presentes na argamassa. Nesta pesquisa o "esqueleto" formado pelos agregados (areia e sedimento), em conjunto com o aglomerante, resultou melhorias desses parâmetros. A absorção de água foi maior para a argamassa de referência ao longo do tempo (Figura 4c). Acredita-se que a estrutura porosa formada se constituiu em uma barreira natural que, fisicamente, dificultou o transporte desse fluido (água), tornando a argamassa menos permeável.

A resistência de aderência foi significativamente maior para a argamassa com sedimento, quando comparada com a argamassa de referência, e com rupturas predominantes do tipo coesivas (Figura 5a).

Nesse sentido, os maiores valores de resistência de aderência, obtidos para a argamassa com sedimento, podem estar relacionados a um melhor empacotamento da mistura e à formação de uma rede de poros que propiciou uma deposição mais homogênea dos produtos de hidratação. Esse resultado veio corroborar com as demais propriedades mecânicas avaliadas nesta pesquisa, em que a argamassa com sedimento obteve desempenho superior ao da argamassa de referência.

Figura 3 - Propriedades das argamassas no estado fresco - (a) índice de consistência, em (b) densidade de massa e (c) teor de ar incorporado
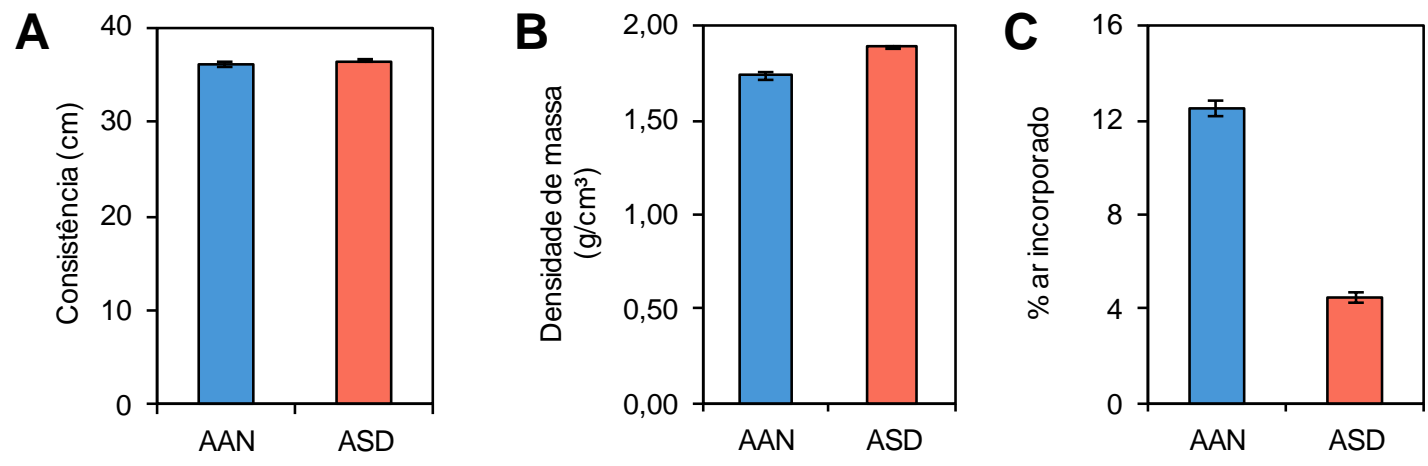

324 Gomes-Pimentel, M.; Paes, I. N. L. 
Figura 4 - Propriedades no estado endurecido - (a) resistência à compressão, (b) resistência à tração na flexão e (c) absorção de água capilar
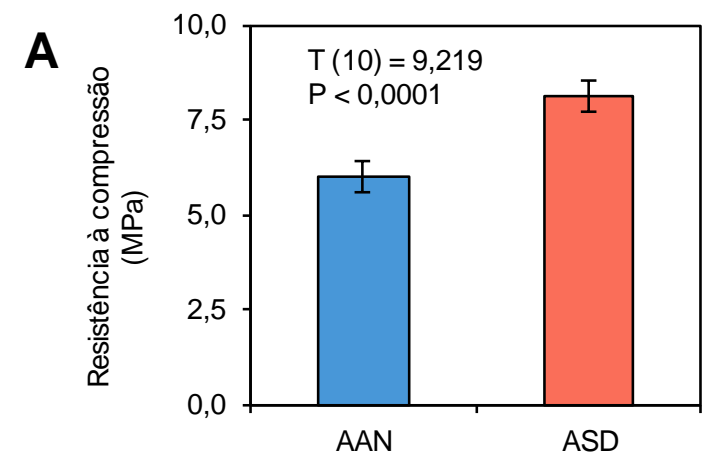

C

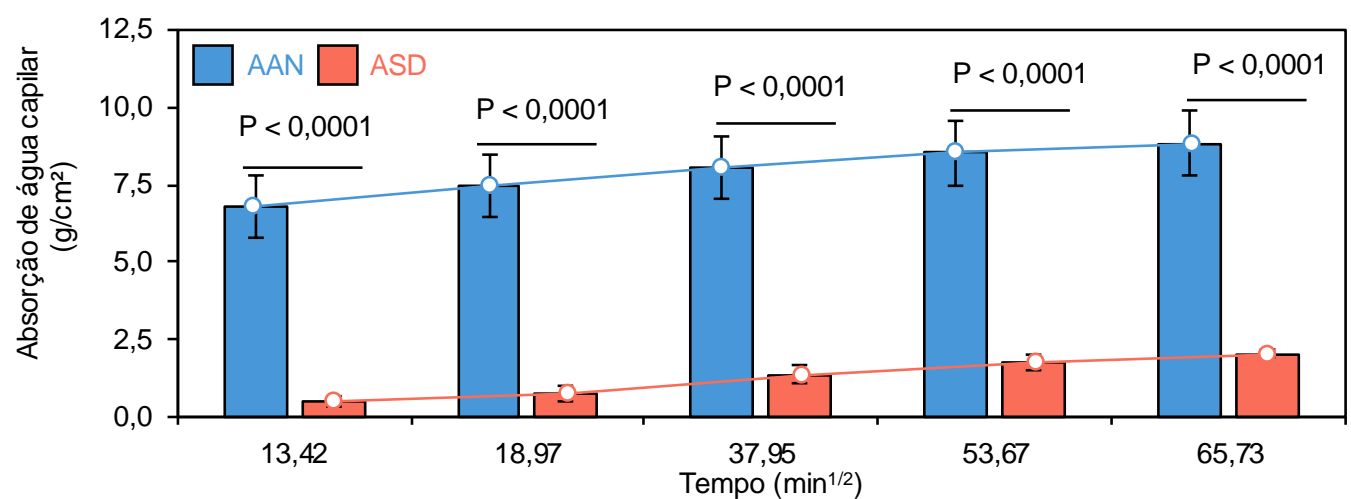

Além disso, o aumento dessa propriedade provavelmente pode ter sido ocasionado pela formação de tobermorita, um composto hidratado de composição química similar ao C-S-H, e de cristais de stratlingita (2 $\left.\mathrm{CaO} . \mathrm{SiO}_{2} \mathrm{Al}_{2} \mathrm{O}_{3} .8 \mathrm{H}_{2} \mathrm{O}\right)$, um filossilicato hexagonal com clivagem paralela perfeita (0001) e uma camada basal com espaçamento de $12,5 \AA$, sendo esse cristal (stratlingita) identificado somente na argamassa com sedimento, por meio de DRX (Figura $5 b$ ).

A argamassa de referência (AAN) apresentou em seu difratograma halo amorfo no intervalo de $2 \theta$ de $21,5^{\circ}$ a $22,5^{\circ}$ e de $23,5^{\circ}$ a $26^{\circ}$, enquanto a argamassa com sedimento (ASD), no mesmo intervalo, apresenta a formação de material cristalino, sugerindo maior estabilidade em longo prazo. Dos cristais semelhantes formados na AAN e ASD, somente os de portlandita apresentaram alteração no tamanho, sendo menor para a argamassa ASD com distância interplanar de 2,56 ̊ (011), quando comparada com a AAN $(2,62 \AA(011))$.

Essa menor dimensão da portlandita formada na argamassa ASD pode ter ocasionado uma densificação de sua microestrutura e que se refletiu no valor mensurado da resistência de aderência. Elucubrando-se ainda a esse respeito, como a portlandita é um cristal com tendência a lixiviar-se em ambientes que porventura estejam sujeitos a um ataque de águas agressivas (ácidas), a menor dimensão desse cristal pode tornar a argamassa mais estável frente a essa envoltória. Ressalta-se, no entanto, que há necessidade de ensaios relacionados à durabilidade do material que comprove tal fato.

A análise de EDS realizada em vários pontos nas argamassas indicou a formação heterogênea de C-A-S-H, além de C-S-H (Figura 5c), principais produtos de hidratação em cimentos que substituem parte do clínquer por material pozolânico. Esses compostos heterogêneos provavelmente refletem a disponibilidade de $\mathrm{Al}^{3+} \mathrm{e}$ $\mathrm{Si}^{4+}$ nos locais de nucleação do gel, por isso a ASD formou C-A-S-H mais ricas em alumínio, possivelmente devido ao sedimento apresentar maiores reservas de $\mathrm{Al}$ (Tabela 1).

Outros fatores como granulometria dos agregados podem ter contribuído para alterar a microestrutura das argamassas, sendo que a AAN apresentou uma microestrutura com poros da ordem de $75 \mu \mathrm{m}$ (Figura 5d) e a formação densa de cristais de calcita depositados nos poros (Figura 5e).

A ocorrência da formação desses cristais é comum em ambientes com reservas de $\mathrm{Ca}(\mathrm{OH})_{2}, \mathrm{CO}_{2}$ e umidade para formar $\mathrm{O} \mathrm{CCO}_{3}$. Como os painéis com as argamassas ficaram expostos às condições naturais, a difusão 
do $\mathrm{CO}_{2}$ atmosférico foi favorecida na argamassa AAN em virtude dos poros maiores. Já a argamassa ASD apresentou uma microestrutura densa com evidentes ligações epitáxicas na zona de transição (Figura 5f).

Essa região é rica em compostos de C-A-S-H e estruturas de stratlingita (Figura 5g). A coalescência de C-AS-H e cristalização de stratlingita podem produzir maior tenacidade e resistência à tração (JACKSON et al., 2014) na argamassa com sedimento. Os cristais de stratlingita na argamassa se assemelham a microfibras que são adicionadas na matriz de concreto e argamassas para aumentar resistências mecânicas como tenacidade e resistência à flexão, com a diferença que os cristais de stratlingita cristalizam-se preferencialmente nas zonas interfaciais, componente mais vulnerável da estrutura de concretos e argamassas (HERNÁNDEZ-CRUZ et al., 2014).

Um mapa realizado por EDS para os espectros de $\mathrm{Si}$, $\mathrm{Ca}$ e $\mathrm{Al}$ em áreas microestruturais reforçam as evidências da formação de coalescência de C-A-S-H e stratlingita na região interfacial da argamassa ASD.

As combinações dos elementos mapeados da argamassa AAN (Figura 6a) mostram a distribuição homogênea da composição de $\mathrm{Si}, \mathrm{Ca}$ e $\mathrm{Al}$ em volta de uma partícula do agregado. Enquanto as combinações dos elementos supracitados na argamassa ASD mostram núcleos ricos em Al na região de interface do sedimento com a matriz (Figura 6b).

Figura 5 - Desempenho e microestrutura das argamassas - (a) resistência à tração, (b) difração de raios $X,(c)$ relações atômicas dos elementos por EDS, (d, e, f e g) microestruturas das argamassas
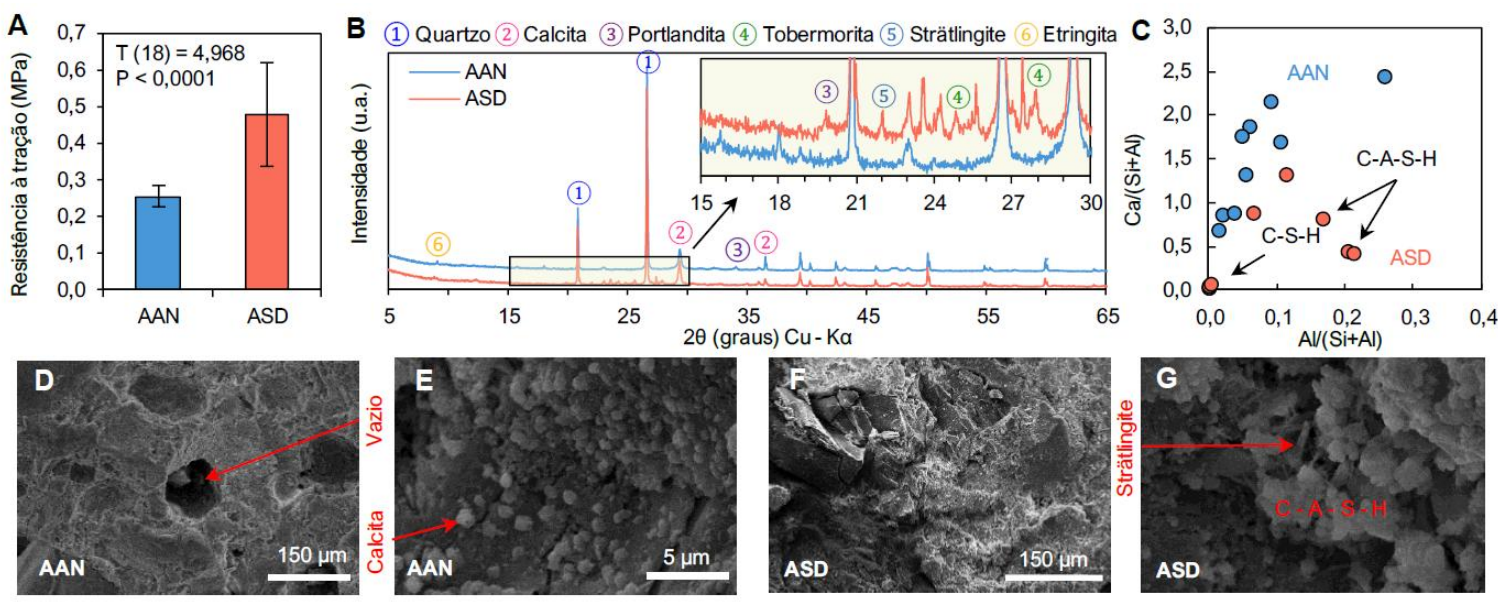

Figura 6 - Microestrutura e mapeamentos dos elementos silício (Si), cálcio (Ca) e alumínio (Al) por EDS (a) argamassa de referência, (b) argamassa com sedimento
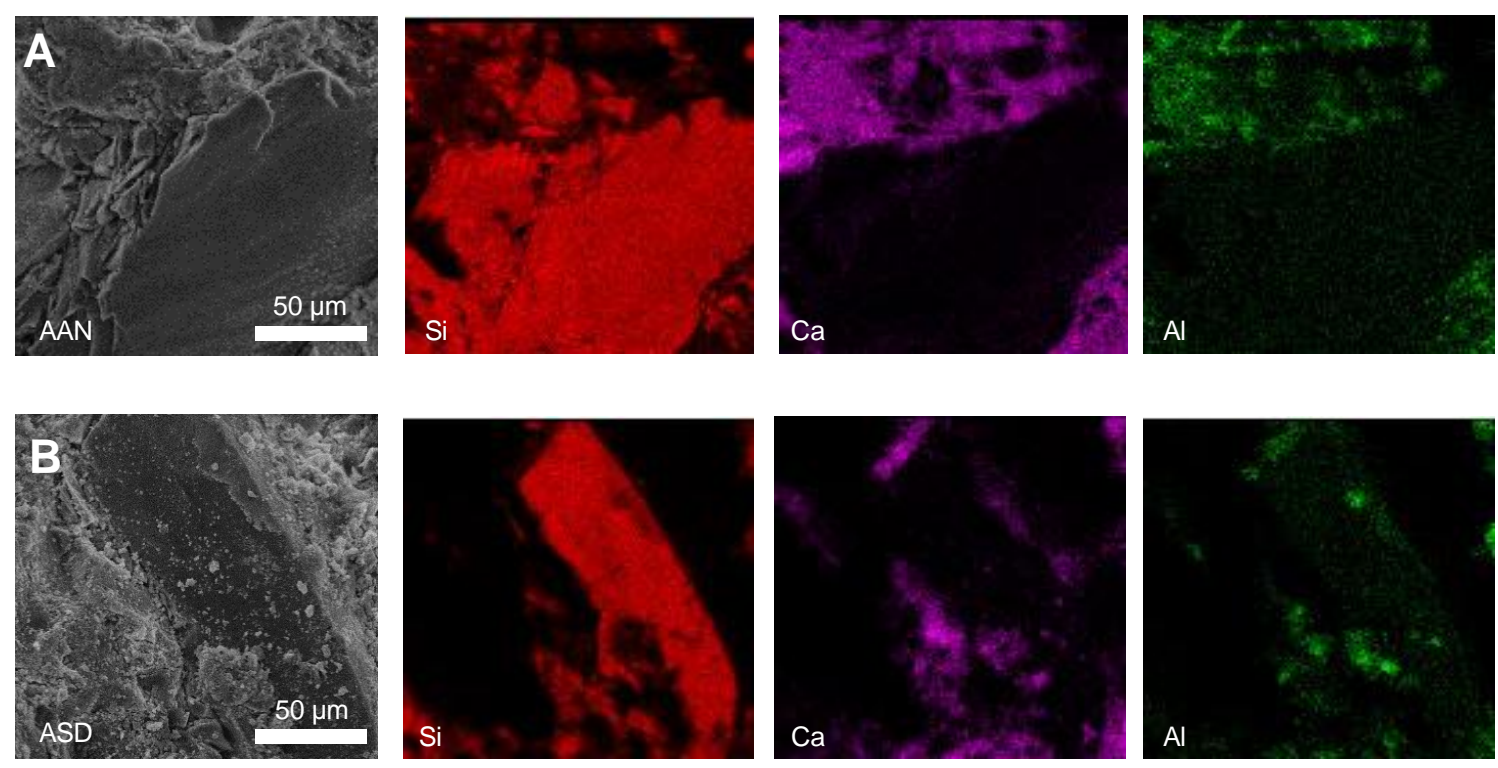

326 Gomes-Pimentel, M.; Paes, I. N. L. 
Assim, os resultados mostram que o sedimento do Rio Amazonas é um agregado promissor para utilização em argamassas de revestimentos. Sua composição mineralógica, especialmente a presença de argilominerais, proporciona a formação de estruturas cristalinas nas argamassas que, por sua vez, podem vir a ocasionar estabilidade em longo prazo aos sistemas de revestimentos. Destaca-se que outras pesquisas já mostraram seu potencial de utilização como agregado de construção ou como material suplementar ao cimento Portland, após tratamento térmico (PIMENTEL; ARAÚJO; PAES, 2017).

Assim, por meio de uma "holística" mais tecnológica, porém embasada em mensurações de base científica, vê-se que a utilização de sedimentos de rios como agregados em concretos e argamassas gera uma alternativa para as localidades que possuem uma extensa bacia hidrográfica, composta por inúmeros rios navegáveis, e que necessitam ser dragados. Estima-se que suas barragens retiveram mais de 100 bilhões de toneladas de sedimentos (MCCARNEY-CASTLE; VOULGARIS; KELTNER, 2010), ao longo do último meio século. Assim, o emprego desse material pode trazer implicações, inclusive de alterações de custos para as obras de infraestruturas que possuem abundância dele (sedimentos).

\section{Conclusões}

A seguir são apresentadas, com base nos resultados do programa experimental e das análises realizadas, as conclusões da pesquisa:

(a) investigaram-se as alterações ocasionadas pela utilização do sedimento do Rio Amazonas como substituto parcial do agregado natural, em argamassas de revestimento. As análises mostraram diferenças relevantes relacionadas à formação microestrutural dos compostos e que alteraram a porosidade da argamassa, ao se inserir o sedimento em sua composição;

(b) a maior cristalinidade dessa argamassa e principalmente os cristais de stratlingita e tobermorita se refletiram em maiores valores mensurados na propriedade de resistência de aderência à tração, quando comparada a uma argamassa de referência. Além disso, a referida propriedade pode ter sido incrementada pela presença de argilominerais, que proporcionam a formação de estruturas cristalinas na região interfacial da argamassa, densificando-a, e, ainda, proporcionar estabilidade química, em longo prazo, para os sistemas de revestimentos; e

(c) assim, com uma abrangência tecnológica e científica, os resultados revelam que o sedimento do Rio Amazonas se apresenta como um agregado promissor para utilização em argamassas de revestimentos.

\section{Referências}

ADEGOLOYE, G. et al. Mineralogical composition of EAF slag and stabilised AOD slag aggregates and dimensional stability of slag aggregate concretes. Construction and Building Materials, v. 115, p. 171$178,2016$.

ASSOCIAÇÃO BRASILEIRA DE NORMAS TÉCNICAS. NBR NM 13528: revestimento de paredes e tetos de argamassas inorgânicas: determinação da resistência de aderência à tração. Rio de Janeiro, 2010.

ASSOCIAÇÃO BRASILEIRA DE NORMAS TÉCNICAS. NBR NM 9779: argamassa e concreto endurecidos: determinação da absorção de água por capilaridade. Rio de Janeiro, 2005c.

ASSOCIAÇÃO BRASILEIRA DE NORMAS TÉCNICAS. NBR NM 13276: argamassa para assentamento e revestimento de paredes e tetos: determinação do índice de consistência. Rio de Janeiro, 2016.

ASSOCIAÇÃO BRASILEIRA DE NORMAS TÉCNICAS. NBR NM 13278: argamassa para assentamento e revestimento de paredes e tetos: determinação da densidade de massa e do teor de ar incorporado. Rio de Janeiro, 2005a.

ASSOCIAÇÃO BRASILEIRA DE NORMAS TÉCNICAS. NBR NM 13279: argamassa para assentamento e revestimento de paredes e tetos: determinação da resistência à tração na flexão e à compressão. Rio de Janeiro, 2005b.

BENDIXEN, M. et al. Time is running out for sand. Nature, v. 571, n. 7763, p. 29-31, 2019.

BENZERZOUR, M.; AMAR, M.; ABRIAKA, N. New experimental approach of the reuse of dredged sediments in a cement matrix by physical and heat treatment. Construction and Building Materials, $v$. 140, p. 432-444, jun. 2017. 
COUVIDAT, J. et al. Feasibility of the reuse of total and processed contaminated marine sediments as fine aggregates in cemented mortars. Construction and Building Materials, v. 112, p. 892-902, jun. 2016.

FONTES, W. C. et al. Mortars for laying and coating produced with iron ore tailings from tailing dams. Construction and Building Materials, v. 112, p. 988-995, 2016.

GENSAC, E. et al. Seasonal and inter-annual dynamics of suspended sediment at the mouth of the Amazon river: The role of continental and oceanic forcing, and implications for coastal geomorphology and mud bank formation. Continetal Shelf Research, v. 118, p. 49-62, 2016.

GUO, Y. et al. Utilization of unprocessed steel slag as fine aggregate in normal- and high-strength concrete. Construction and Building Materials, v. 204, p. 41-49, 2019.

HERNÁNDEZ-CRUZ, D. et al. Multiscale characterization of chemical-mechanical interactions between polymer fibers and cementitious matrix. Cement and Concrete Composites, v. 48, p. 9-18, 2014.

JACKSON, M. D. et al. Resilience of Imperial Roman architectural mortar. Proceedings of the National Academy of Sciences, v. 111, p. 18484-18489, 2014.

KHYALIYA, R. K.; KABEER, K. I. S. A.; VYAS, A. K. Evaluation of strength and durability of lean mortar mixes containing marble waste. Construction and Building Materials, v. 147, p. 598-607, 2017.

KOEHNKEN, L. Impacts of sand mining on ecosystem structure, process e biodiversity in rivers. London: WWF, 2018.

LAOUFI, L.; SENHADJIA, Y.; BENAZZOUKB, A. Valorization of mud from Fergoug dam in manufacturing. Case studies in construction materials, v. 5, p. 26-38, 2016.

MCCARNEY-CASTLE, K.; VOULGARIS, G.; KETTNER, A. J. Analysis of Fluvial Suspended Sediment Load Contribution through Anthropocene History to the South Atlantic Bight Coastal Zone, U.S.A. The Jornal of Geology, v. 118, p. 399-416, 2010.

PARK, E.; LATRUBESSE, E. M. Modeling suspended sediment distribution patterns of the Amazon River using MODIS data. Remote Sensing of Environment, v. 147, p. 232-242, may 2014.

PEDUZZI, P. Sand, rarer than one thinks Article reproduced from United Nations Environment Programme (UNEP) Global Environmental Alert Service (GEAS). Environmental Development, v. 11, p. 208-218, 2014.

PIMENTEL, M. G.; ARAUJO, J.; PAES, I. N. L. Propriedades mecânicas e de durabilidade de argamassas de revestimento com substituição parcial do agregado miúdo por sedimento do rio amazonas. In: SIMPÓSIO BRASILEIRO DE TECNOLOGIA DAS ARGAMASSAS, 12., São Paulo, 2017. Anais [...] São Paulo: USP, 2017.

PIMENTEL, M. G.; PAES, I. N. L. Análise microestrutural de argamassa de revestimento com substituição parcial do agregado natural por sedimento do rio amazonas. In: SIMPÓSIO BRASILEIRO DE TECNOLOGIA DAS ARGAMASSAS, Goiânia, 2019. Anais [...] Goiânia, 2019.

REGE, A. Not biting the dust: using a tripartite model of organized crime to examine India's Sand Mafia. International Journal of Comparative and Applied Criminal Justice, v. 40, n. 2, p. 101-121, 2016.

ROZIÈREA, E. et al. Valorisation of sediments in self-consolidating concrete: Mix-design and microstructure. Construction and Building Materials, v. 81, p. 1-10, apr. 2015.

SUTHERLAND, W. J. et al. A 2017 horizon scan of emerging issues for global conservation and biological diversity. Trends in Ecology and Evolution, v. 32, n. 1, p. 31-40, 2017.

SVERDRUP, H. U.; KOCA, D.; SCHLYTER, P. A simple system dynamics model for the global production rate of sand, gravel, crushed rock and stone, market prices and long-term supply embedded into the WORLD6 model. BioPhysical Economics and Resource Quality, v. 2, n. 2, 2017.

UNITED NATIONS ENVIRONMENT PROGRAMME. Global material flows and resource productivity: assessment report for the UNEP international resource panel. Paris, 2016. Relatório.

WANG, L. et al. Mixture design and treatment methods for recycling contaminated sediment. Journal of Hazardous Materials, v. 283, p. 623-632, fev. 2015. 
YARAGAL, S. C.; BASAVANA GOWDA, S. N.; RAJASEKARAN, C. Characterization and performance of processed lateritic fine aggregates in cement mortars and concretes. Construction and Building Materials, v. 200, p. 10-25, 2019.

BENDIXEN, M. et al. Time is running out for sand. Nature, v. 571, n. 7763, p. 29-31, 2019.

BENZERZOUR, M.; AMAR, M.; ABRIAKA, N. New experimental approach of the reuse of dredged sediments in a cement matrix by physical and heat treatment. Construction and Building Materials, $v$. 140, p. 432-444, jun. 2017.

COUVIDAT, J. et al. Feasibility of the reuse of total and processed contaminated marine sediments as fine aggregates in cemented mortars. Construction and Building Materials, v. 112, p. 892-902, jun. 2016.

FONTES, W. C. et al. Mortars for laying and coating produced with iron ore tailings from tailing dams. Construction and Building Materials, v. 112, p. 988-995, 2016.

GENSAC, E. et al. Seasonal and inter-annual dynamics of suspended sediment at the mouth of the Amazon river: the role of continental and oceanic forcing, and implications for coastal geomorphology and mud bank formation. Continetal Shelf Research, v. 118, p. 49-62, 2016.

GUO, Y. et al. Utilization of unprocessed steel slag as fine aggregate in normal- and high-strength concrete. Construction and Building Materials, v. 204, p. 41-49, 2019.

HERNÁNDEZ-CRUZ, D. et al. Multiscale characterization of chemical-mechanical interactions between polymer fibers and cementitious matrix. Cement and Concrete Composites, v. 48, p. 9-18, 2014.

JACKSON, M. D. et al. Resilience of Imperial Roman architectural mortar. Proceedings of the National Academy of Sciences, v. 111, p. 18484-18489, 2014.

KHYALIYA, R. K.; KABEER, K. I. S. A.; VYAS, A. K. Evaluation of strength and durability of lean mortar mixes containing marble waste. Construction and Building Materials, v. 147, p. 598-607, 2017.

KOEHNKEN, L. Impacts of sand mining on ecosystem structure, process e biodiversity in rivers. London: WWF, 2018.

LAOUFI, L.; SENHADJIA, Y.; BENAZZOUKB, A. Valorization of mud from Fergoug dam in manufacturing. Case studies in construction materials, v. 5, p. 26-38, 2016.

LEDESMA, E. F. et al. Properties of masonry mortars manufactured with fine recycled concrete Aggregates. Construction and Building Materials, v. 71, p.289-298, 2014.

MCCARNEY-CASTLE, K.; VOULGARIS, G.; KETTNER, A. J. Analysis of Fluvial Suspended Sediment Load Contribution through Anthropocene History to the South Atlantic Bight Coastal Zone, U.S.A. The jornal of geology, v. 118, p. 399-416, 2010.

PARK, E.; LATRUBESSE, E. M. Modeling suspended sediment distribution patterns of the Amazon River using MODIS data. Remote Sensing of Environment, v. 147, p. 232-242, may 2014.

PEDUZZI, P. Sand, rarer than one thinks Article reproduced from United Nations Environment Programme (UNEP) Global Environmental Alert Service (GEAS). Environmental Development, v. 11, p. 208-218, 2014.

PIMENTEL, M. G.; ARAUJO, J.; PAES, I. N. L. Propriedades mecânicas e de durabilidade de argamassas de revestimento com substituição parcial do agregado miúdo por sedimento do rio amazonas. In: SIMPÓSIO BRASILEIRO DE TECNOLOGIA DAS ARGAMASSAS, 12., São Paulo, 2017. Anais [...] São Paulo: USP, 2017.

PIMENTEL, M. G.; PAES, I. N. L. Análise microestrutural de argamassa de revestimento com substituição parcial do agregado natural por sedimento do rio amazonas. In: SIMPÓSIO BRASILEIRO DE TECNOLOGIA DAS ARGAMASSAS, Goiânia, 2019. Anais [...] Goiânia, 2019.

REGE, A. Not biting the dust: using a tripartite model of organized crime to examine India's Sand Mafia. International Journal of Comparative and Applied Criminal Justice, v. 40, n. 2, p. 101-121, 2016.

ROZIÈREA, E. et al. Valorisation of sediments in self-consolidating concrete: mix-design and microstructure. Construction and Building Materials, v. 81, p. 1-10, apr. 2015.

SUTHERLAND, W. J. et al. A 2017 horizon scan of emerging issues for global conservation and biological diversity. Trends in Ecology and Evolution, v. 32, n. 1, p. 31-40, 2017. 
SVERDRUP, H. U.; KOCA, D.; SCHLYTER, P. A simple system dynamics model for the global production rate of sand, gravel, crushed rock and stone, market prices and long-term supply embedded into the WORLD6 model. BioPhysical Economics and Resource Quality, v. 2, n. 2, 2017.

UNITED NATIONS ENVIRONMENT PROGRAMME. Global material flows and resource productivity: assessment report for the UNEP international resource panel. Paris, 2016. Relatório.

WANG, L. et al. Mixture design and treatment methods for recycling contaminated sediment. Journal of Hazardous Materials, v. 283, p. 623-632, fev. 2015.

YARAGAL, S. C.; BASAVANA GOWDA, S. N.; RAJASEKARAN, C. Characterization and performance of processed lateritic fine aggregates in cement mortars and concretes. Construction and Building Materials, v. 200, p. 10-25, 2019.

ZHAO, Z. et al. Influence of fine recycled concrete aggregates on the properties of mortars. Construction and Building Materials, v. 81, p. 179-186, 2015.

\section{Agradecimentos}

Os autores agradecem ao Laboratório de Microanálise do Instituto de Geociências da Universidade Federal do Pará (UFPA) pela ajuda na análise microestrutural, ao Museu de. Geociências (MUGEO) da UFPA pela análise granulométrica a laser do sedimento, ao Laboratório de Raios X, Análise Termica e Dielétrica do Programa de Pós-Graduação em Fisica da UFPA pela análise de DRX, ao Laboratório de Engenharia Civil do Instituto de Tecnologia da UFPA pelos ensaios mecânicos e finalmente ao Grupo de Pesquisa de Materiais de Construção Civil (GPMAC) da UFPA pelos constantes debates e contribuições para construção do presente artigo.

Maurílio Gomes-Pimentel

Instituto de Tecnologia | Universidade Federal do Pará | Rua Augusto Corrêa, 01 | Belém -PA - Brasil | CEP 66075-110 | Tel.: (91) 980301394 | E-mail: mauriliogpimentel@gmail.com

Isaura Nazaré Lobato Paes

Instituto de Tecnologia | Universidade Federal do Pará | E-mail: isaurapaes@ufpa.br

\section{Ambiente Construído}

Revista da Associação Nacional de Tecnologia do Ambiente Construído

Av. Osvaldo Aranha, 99 - $3^{\circ}$ andar, Centro

Porto Alegre - RS - Brasil

CEP $90035-190$

Telefone: +55 (51) 3308-4084

Fax: +55 (51) 3308-4054

www.seer.ufrgs.br/ambienteconstruido

E-mail: ambienteconstruido@ufrgs.br 